\title{
Seismic Probabilistic Risk Assessment and its Application to Utility Safety Decisions
}

\author{
J.-S. Wu* \\ University of California, Los Angeles, CA USA \\ F. L. Yu, P. C. Chen, E. Lin \\ Taiwan Power Company, Taipei, Taiwan
}

\section{INTRODUCTION.}

One of the active projects of TPC in recent years is completion by 1990 of probabilistic risk assessments (PRA) for the three nuclear power plants it currently owns. To this date, PRA's have been completed for two of the three -- Kuosheng, a boiling-water mode1 6 reactor (BWR-6) with a Mark III containment; and Maanshan, a pressurized-water reactor (PWR) with a large dry containment. A similar study for the third plant -- Chinsan, a BWR-4 with a Mark I containment -- is currently underway. The Kuosheng PRS (Taiwan Power Company, 1985) and Chinsan PRA include both an inplant analysis (Level I PRA) and a containment analysis (Level II PRA), while the Maanshan PRA (Taiwan Power Company, 1987) is a Level I PRA only.

Taiwan is located in a region of high seismicity. Although the three nuclear power plants currently owned by TPC are all designed at a relatively high level of safe shutdown earthquake (SSE), $0.4 \mathrm{~g}$, impacts on plant safety from seismic-induced accidents are still of great concern to the utility managers. As a result, seismic risk analyses are key issues on both Kuosheng and Maanshan PRA's, and detailed analyses on seismic-induced events are performed. The main objective of this paper is to discuss some specific features of the results of these studies as well as some of the difficulties of implementing recommendations which utility managers are most likely to face at plant sites in areas of high seismicity, such as those at TPC. Although the results of seismic PRAs reflect the best knowledge of the analysts within the range of data and resources that were available to them, the broad distributions of the results create a very difficult premise in the decision-making process for utility managers.

II. DISCUSSION OF THE RESULTS OF KUOSHENG AND MAANSHAN SEISMIC PRA'S.

Taiwan is a member of the Ryukyu-Taiwan-Philippine island arc chain, which rims the western border of the Pacific Ocean. Tectonically, most of the island of Taiwan is located on the eastern border of the Eurasian Plate; and the Eastern Coastal Range of Taiwan is on the western edge of the Philippine Sea Plate. The narrow linear Eastern Longitudinal Valley lies between, forming the collision boundary between the converging plates. Accordingly, much of Taiwan's geological structures and tectonic features have resulted from collisions between the plates from the end of the Pleistocene age until the present.

This paper is adapted from another paper sent to the Reliability Engineering and System Safety by the same authors.

*Current address: Mechanical, Aerospace and Nuclear Engineering Department, UCLA, Los Angeles, California 90024. 
Kuosheng and Chinsan are located at the northern tip of Taiwan, and Maanshan is at the southern tip. The geological and tectonic environment indicates that these three plant sites are located in regions of high seismicity.

In Kuosheng seismic PRA, the three-zoning scheme and three-attenuation equations model used in the analysis results in a set of nine possible hazard curves for the site. This is shown in Figure 1. The tectonic location and the geological structure around Maanshan are similar to those of Kuosheng; the two sites are not too far apart. It is not surprising that the hazard curves for the two sites are very similar. There is one point worth noting, however; that according to seismology records, the distances between Maanshan site and the earthquake epicenters surrounding it are closer than those at Kuosheng, and the resulting Maanshan hazard curves are more sensitive to the forms of attenuation models. The process in achieving consensus about the appropriateness of the attenuation models and the set of weights among a group of experts, in the case of Maanshan, is much more roundabout and leaves more room for dispute.

Several observations are made on the resulting hazard curves and their impact on total plant risk. They are discussed in the following paragraphs.

The resulting hazard curves shown in Figure 1 indicate that the exceeding frequency of occurrence for plants in Taiwan are, in general, an order of magnitude higher than those for plants in the eastern United States; e.g., Zion (Commonwealth Edison Company, 1981), where geological structures are considered rather stable. In Figure 2, however, if the plant SSE is used as the scale for effective ground acceleration instead of in terms of $g^{\prime} s$, the difference diminishes between the two groups of hazard curves (the curves shown in Figure 2 are the highest exceeding frequency curve in each group), especially for higher ground motion accelerations. Since all safety-related structures, systems, and components are designed according to design basis earthquakes, or one-half SSE, the general agreement of these two sets of hazard curves based on SSE scale seems to signal a relief for those plants with high seismic activities. There is, however, an issue related to accident sequences caused by the combination of seismic- and nonseismic-induced failures which has been generally overlooked under the current single failure criterion. This point will be made clear after the following discussion.

The safety-related plant structures and components of both Kuosheng and Maanshan are designed at $0.4 \mathrm{~g}$ as their SSE. This is coupled with sophisticated qualification techniques and design codes. The majority of safety-related structures and components under investigation have relatively high ground acceleration capacities. In that sense, the analysis is, in general, confirmed with the results of the SSMRP (Bohn, 1984) study, which is carried out for the plants east of the Rocky Mountains in the United States, that nuclear power plants in those regions enjoy quite comfortable safety margins with repect to seismic events. However, our studies show a shift of dominant risk contributors to the combinations of seismic- and nonseismic-induced failures as the frequencies of the seismological events at the plant sites shift from a low occurrence rate to a high one. At present, the levels of significance of these risk contributors are still unknown.

The failure of equipment in the switchyard which lead to a partial or total loss of offsite power has been identified as the dominant contributor to plant risk in both Kuosheng and Maanshan PRA studies. The equipment in the switchyard is not required to satisfy the seismic qualifications; it is designed at a capacity much lower than one-half SSE, with ceramic insulators identified as the weakest parts. To meet the single failure criterion, the plant's onsite source of emergency AC power is then designed to satisfy all the seismic qualifications. The PRA studies, 
however, identified that accidents involving more than one incident (e.g., a seismic-induced failure of one component followed by a nonseismic-induced failure of another), are also of significance. This is especially true when the plant under investigation is located in an area of high seismicity, where seismic-induced events occur more frequently.

Unlike the case for a plant at site where seismic events are insignificant, one of the dominant risk contributors for the station blackout at site of high seismicity comes from the seismic-induced loss of offsite power, followed by a nonseismic-induced failure of the onsite AC power source. If this is the case, the recent studies of station blackout (Baranowsky, 1985), which only emphasize the internal causes, ought to take a new look at those seismic-related events, at least for plants in areas of high seismicity. Introducing uncertainty factors will complicate the risk assessment and make the unrelated decision problems more intricate. This issue will not be discussed here.

For those plants designed only according to the single failure criteria, the above discussion points out that utilities in areas of high seismicity may have expended significant resources in fulfilling the current regulatory requirements of seismic qualifications, while they neglect those coupled failures, from which the true dominant risk comes. These utilities may have not obtained the amount of risk reduction that they would have expected, and their overall design is very likely not to be cost effective. A similar conclusion is obtained by Evans, et al. (1986), where only the mean seismic risks are compared. The results in Figure 3 show that the seismic risk studies of plants on the east coast of the United States, where seismic activity is low, typically have the majority of contribution to risk coming from earthquakes at about two to three times of their SSE's, and these plants therefore enjoy a comfortable safety margin. Were the same conclusion drawn for plants in areas of high seismicity, they would experience the same seismic margin expressed in the SSMRP program. The example shown in Figure 3, however, indicates that for plants at high seismicity sites, the major contributions to core melt frequency come from a much lower range of SSE's and, thus, much more vulnerable to seismic events. It is noted that such vulnerability comes from a combination of nonseismic-induced failures of component and a seismic-induced failure of equipment that does not require seismic qualification and, therefore, is irrelevant to the stringency of the current code of seismic qualification. If the uncertainty of the results of seismic risk analysis can be reduced to an intuitively acceptable level, the information discussed above can be of great use in the design of future plants or in the improvement of existing plants.

\section{DIFFICULTIES OF IMPLEMENTING THE RESULTS OF SEISMIC PRA.}

It is well understood that the analysts attempt to present results of seismic risk analysis which are based on their best available knowledge and data, but which are subject to inevitable limits of time and resources. This section, therefore, addresses the difficulties that utility managers will likely face as they employ these results to aid their decision-making. Because TPC's plants are located in areas of high seismicity, its managerial personnel find it very difficult to deal with the breadth of both the qualitative and quantitative uncertainties which arise at each stage of the seismic PRA. They are also unsure about how the results of seismic PRA should be integrated with the rest of the results of PRA into the decision-making process. Questions pertaining to methodology and to the interpretation and implementation of the results are inevitably raised.

(1) Methodology Overview.

Some general concerns about the methods used to estimate the seismic-induced risk are based on discussion in preceding sections. These concerns may undermine the credibility of the results and leave doubts about 
the effectiveness of the actions recommended by the analysis.

(A) Lack of Supporting Data. It is acknowledged in each step of the seismic risk analysis that the data supporting the analysis are either insufficient or incomplete. In order to be rendered numerically, results are to some extent provisional, ultimately depending on the experts' opinions or analysts' judgments. It is important to recognize that even these experts may not themselves be completely comfortable with the notion of precision which is implied by quantification or with the particular numbers they have had to provide or to work with in any specific situation. The current state-of-the-art PRA has not been successfully shown either the explicit impact of such imprecise data or the reliability of the opinion of the experts. For example, it is pointed out in Kuosheng and Maanshan studies that the existing records are not sufficient to determine fully the occurrence rate and the maximum possible magnitude of the earthquakes around the site. This lack of field information has influenced the validity of the final set of hazard curves, along with the modeling uncertainties and experts' judgment, without specifically presenting the impact from each category of lack of information in the analyses. It would be more informative to the users (in this case, the utility managers) in their decision-making process if the final seismic risk were presented in a way that provides more details about the impact on total seismic risk from each category of uncertain information.

(B) Judgment Used in the Analysis. Experts' judgments are used, implicity and explicitly, throughout the analysis in areas from the definition of failure modes and the choice of models to the assessment of numerical values of parameters. In many cases, the judgment of experts or analysts can be the determining factor for the frequencies of accident sequences being estimated. Formal policy about eliciting and using expert opinions is still lacking. Problems such as aggregation, biases, miscalibration, over-confidence, decomposition during eliciting, and dependency in the knowledge base are barely touched on or mentioned in the analysis, although problems in these areas are well noted (Fischhoff, 1986). In cases when a seismic-induced accident sequence is identified as one of the dominant contributors to total plant risk, and the process of getting its numerical figures relies heavily on expert opinion, more sophisticated approaches that give more thought to the above problem areas will make the results more convincing.

(C) The Treatment of Modeling Uncertainty. Since the physical phenomena are not well understood, uncertainty exists about the appropriateness of the models being used. Some authors have even suggested that in PRA analysis, a major source of uncertainty could be the model itself. Although there are some general discussions about the different kinds of treatment for modeling uncertainties (Parry, 1986), guidance is lacking in the present PRA state-of-the-art methodology concerning how to cope with them. Implicitly and explicitly, different approaches have been adopted at different stages of the analysis. For example, in the seismic hazard analysis, the $n *_{m}$ hazard curves are used to represent the $n$ models of zoning schemes and the $\mathrm{m}$ models of attenuation in ground motion, and a set of weights is assigned to each curve to express the degree of belief over the set of curves; however, in the fragility analysis, modeling uncertainty is implicitly expressed in the parameters B's, blended together with all the other uncertainty factors. Furthermore, although some authors have suggested that the appropriateness of different models should be weighted over the analysts' degree of belief, there are still debates going on about the epistemological interpretation of the "degree of belief". Even for those who find subjective probability interpretation appealing, there exist problems in the eliciting of numerical values. Seismic risk analysis, and possibly some other areas in PRA methodology, need not only investigation of ways to treat the modeling uncertainties, but also more fundamental excursuses on the underlying logic and concepts. 
(2) The Interpretation of Results and Their Implementation in Decision-Making.

Results of a seismic risk analysis presented to the utility managers typically are curves with broad distributions that span several orders of magnitude. Because these curves usually have long tails at the end of high consequences on the logrithmic scale, the mean values of these distributions are of ten numerically equivalent to their higher percentiles, sometimes even higher than the nineties. Unlike those cases resulting for internal events, the median and mean values of these seismic-risk distributions are ususally quite separated in their numerical values, sometimes differing by orders of magnitude. Since there is still no theoretical framework to justify the use of the mean, the median, or other characteristics of the distribution -- percentiles, moments, etc. -- as an index to rank the risk of the accident sequences involved, the difference in the width of the distributions makes the ranking process especially difficult when accident sequences involving seismic and internal events are evaluated together. Significantly different rankings among sequences may result from using different characteristic values -- mean or median, for example -- as risk index. This raises the problem of how to interpret the resulting risk distributions and how to make decisions in the face of uncertainties, especially for those cases in which some of the distributions may be broad. This issue is certainly not new; however, it remains an area that is essential to the utility in decision-making despite its continuing lack of a satisfactory solution. If the results of the studies identify one group of components rather than another as being critical to the improvement of plant safety, then the utility will invest significant resources to improve a completely different list of equipment. This, in turn, will have different realistic and concrete impacts on plant safety.

The PRA analysts presume that the information contained in a PRA study may find a suitable role in the decision-making process and that all factors will be somehow synthesized to produce the optimum decision. However, under current circumstances, for the owners of the plants (at least of plants located in areas of high seismicity), decisions remain very difficult for many reasons. The distributions are broadly spanned. The risk index is confused. The expertise required to extrapolate any meaningful intuition that can be practically applied to the decision-making process from the technical conclusions is usually too sophisticated to be easily grasped by persons outside the specialty. Finally, the general practice and norms for making decisions under uncertainty are not well established. Such problems as these continue to make the application of the results of seismic risk analysis fraught with problems.

As a result, despite the current best intentions of utilities to apply the results of seismic PRA to the decisions related to the safety of nuclear power plants, the state-of-the-art is unfortunately still too premature for us to present any optimistic conclusions.

\section{REFERENCES}

Baranowsky, P.W., Evaluation of Station Blackout Accidents at Nuclear Power Plants, NUREG1302, USNRC, 1985.

Bohn, M.P., et al., Application of the SSMRP Methodology to the Seismic Risk at the Zion Nuclear Power Plant, NUREG/CR-3428, USNRC, 1984 .

Commonwealth Edison Company, Zion Probabilistic Safety Study, 1981.

Evans, M.G.K., Parry, G.W., and Wu, J.S., "The Treatment of a Seismically Induced Loss of Offsite Power Initiating Event in Probabilistic Risk Assessment", presented at the International ANS Meeting on Thermal Reactor Safety, San Diego, 1986, pp. VIII.2, 1-8.

Fischhoff, B., "Decision-Making in Complex Systems", In: Intelligent Decision Support in Process Environments, E. Hollnagel, G. Mancini, and D.D. Woods (eds)., Springer-Verlag, NY, 1986, pp. 61-85.

Parry, G.W., "A Discussion on the Use of Judgment in Representing Uncertainty in PRAs", Nucl. Eng. Design, 93 (1986), pp. 135-144.

Taiwan Power Company, Kuosheng Probabilistic Risk Assessment, 1985.

Taiwan Power Company, Maanshan Probabilistic Risk Assessment, 1987. 


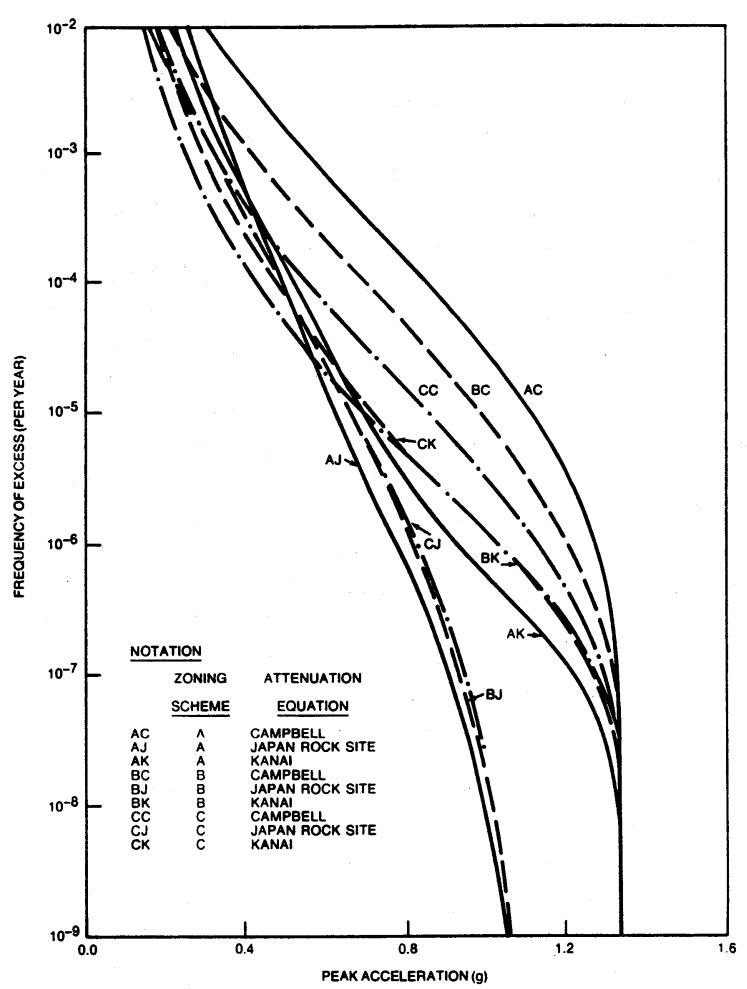

FIGURE 1.

POSSIBLE SEISMIC HAZARD CURVES FOR KUOSHENG SITE

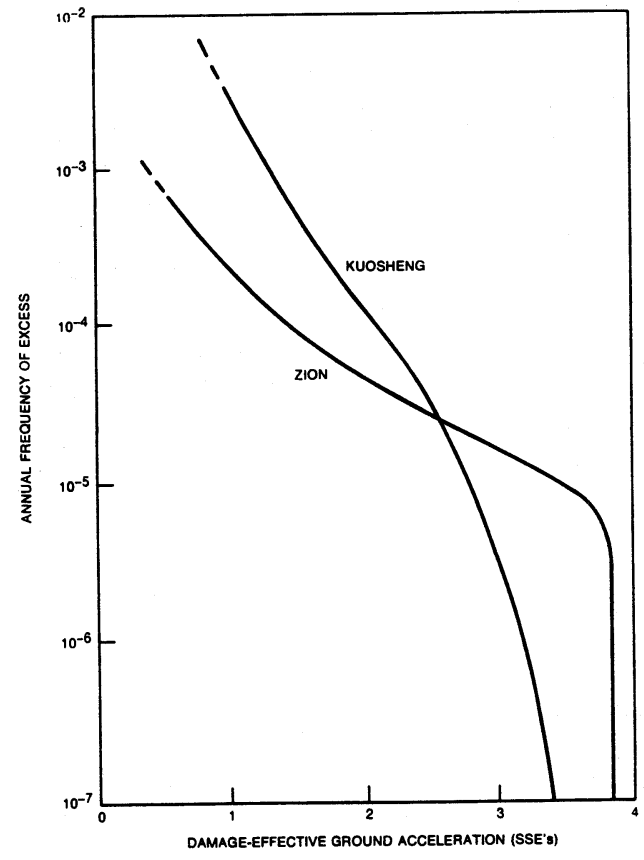

FIGURE 2

SEISMIC HAZARD CURVES FOR KUOSHENG AND ZION SITE IN SSE'S

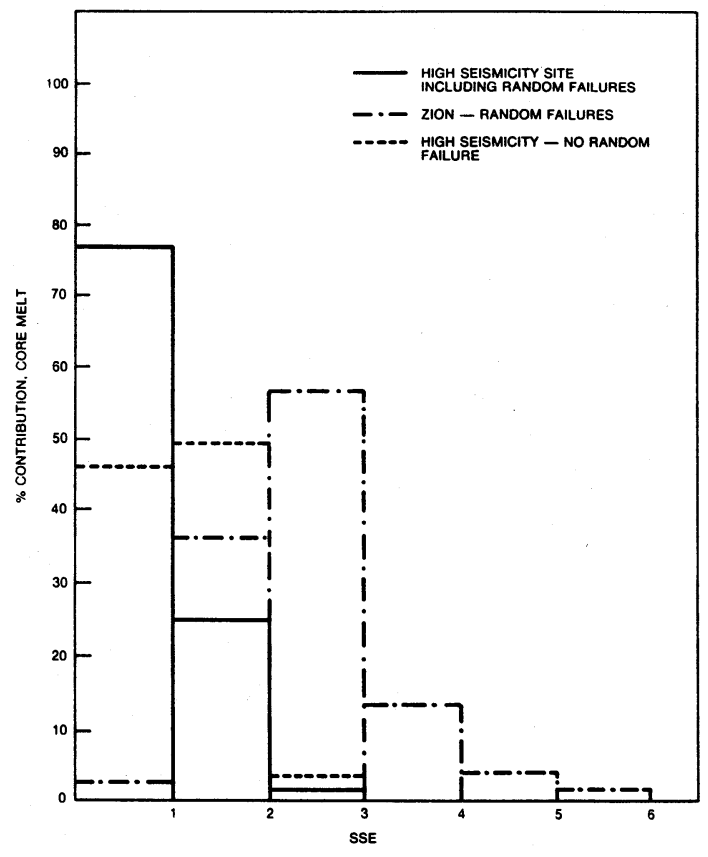

FIGURE 3.

CONTRIBUTION OF RANGES OF GROUND ACCELERATION TO CORE MELT FREQUENCY 